\title{
Reduced Complexity MMSE Detection for BLAST Architectures
}

\author{
Ronald Böhnke, Dirk Wübben, Volker Kühn, and Karl-Dirk Kammeyer \\ Department of Communications Engineering \\ University of Bremen \\ Otto-Hahn-Allee \\ D-28359 Bremen, Germany \\ Email: \{boehnke,wuebben,kuehn,kammeyer\}@ant.uni-bremen.de
}

\begin{abstract}
Theoretical and experimental studies have shown that layered space-time architectures like the BLAST system can exploit the capacity advantage of multiple antenna systems in rich-scattering environments. In this paper, we present a new efficient algorithm for detecting such architectures with respect to the MMSE criterion. This algorithm utilizes a sorted QR decomposition of the channel matrix and leads to a simple successive detection structure. The algorithm needs only a fraction of computational effort compared to the standard V-BLAST algorithm and achieves the same bit error performance.

Index Terms-BLAST, MIMO systems, Zero-Forcing and MMSE detection, wireless communication.
\end{abstract}

\section{INTRODUCTION}

In rich-scattering environments the use of multiple antenna systems provides an enormous increase in spectral efficiency compared to single antenna systems [1]. A multiple-input multiple-output (MIMO) system that exploits this potential is the V-BLAST (Vertical Bell Labs Layered Space-Time) architecture proposed in [2]. It uses a vertically layered coding structure, where independent code blocks (called layers) are associated with a particular transmit antenna. At the receiver, these layers are detected by a successive interference cancellation technique which nulls the interferers by linearly weighting the received signal vector with a zero-forcing nulling vector (ZF-BLAST). A very efficient detection algorithm utilizing the QR decomposition of the channel matrix was proposed by the authors in [3], [4]. It jointly calculates an optimized detection order and the $\mathrm{QR}$ decomposition of the channel matrix and is called ZF-SQRD (ZF Sorted QR Decomposition). An adaption of the original ZF-BLAST to the MMSE criterion was presented in [5] and a version with lower complexity was introduced in [6].

In this paper, we propose an extension of the ZF-SQRD algorithm to the MMSE solution called MMSE-SQRD. As it does not always find the optimal detection order, a performance degradation may occur. If this drawback is not acceptable for the specific application, MMSE-SQRD can be used as a pre-ordering for the optimal strategy, leading to the ideal detection sequence with reduced computational effort.

This work was supported in part by the German ministry of education and research (BMBF) under grant $01 \mathrm{BU} 153$.
The remainder of this paper is organized as follows. In Section II, the system model is introduced. In Section III, several ZF detection algorithms are reviewed. MMSE extensions of these detection algorithms are investigated and the new MMSE-SQRD is described in Section IV. The performances of the different methods are compared in Section V and concluding remarks can be found in Section VI.

\section{SYSTEM DESCRIPTION}

We consider a multiple antenna system with $n_{T}$ transmit and $n_{R} \geq n_{T}$ receive antennas as shown in Fig. 1. The data is demultiplexed into $n_{T}$ substreams of equal length (called layers). These substreams are mapped onto $M$-PSK or $M$ QAM symbols $s_{1}, \ldots, s_{n_{T}}$ and simultaneously transmitted over the $n_{T}$ antennas.

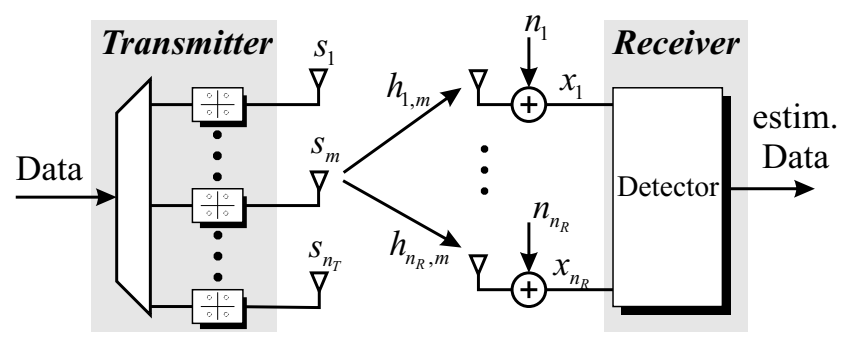

Fig. 1. Model of a MIMO system with $n_{T}$ transmit and $n_{R}$ receive antennas.

In order to describe the MIMO system, one time slot of the time-discrete complex baseband model is investigated. Let $^{1} \mathbf{s}=\left[\begin{array}{lll}s_{1} & \ldots & s_{n_{T}}\end{array}\right]^{T}$ denote the $n_{T} \times 1$ vector of transmit symbols, then the corresponding $n_{R} \times 1$ receive signal vector $\mathbf{x}=\left[x_{1} \ldots x_{n_{R}}\right]^{T}$ is given by

$$
\mathbf{x}=\mathbf{H s}+\mathbf{n} .
$$

In (1), $\mathbf{n}=\left[n_{1} \ldots n_{n_{R}}\right]^{T}$ represents the white gaussian noise of variance $\sigma_{n}^{2}$ observed at the $n_{R}$ receive antennas while the

${ }^{1}$ Throughout this paper, $(\cdot)^{T}$ and $(\cdot)^{H}$ denote matrix transposition and hermitian transposition, respectively. Furthermore $\mathbf{I}_{\alpha}$ indicates the $\alpha \times \alpha$ identity matrix and $\mathbf{0}_{\alpha, \beta}$ denotes the $\alpha \times \beta$ all zero matrix. 
average transmit power of each antenna is normalized to one, i.e.

$$
\mathrm{E}\left\{\mathbf{s s}^{H}\right\}=\mathbf{I}_{n_{T}} \quad \text { and } \quad \mathrm{E}\left\{\mathbf{n n}^{H}\right\}=\sigma_{n}^{2} \mathbf{I}_{n_{R}} .
$$

The $n_{R} \times n_{T}$ channel matrix $\mathbf{H}$ contains uncorrelated complex gaussian fading gains with unit variance. We assume a flatfading environment, where the channel matrix $\mathbf{H}$ is constant over a frame and changes independently from frame to frame (block fading channel). The distinct fading gains are assumed to be perfectly known by the receiver.

In order to detect the transmit signals at the receiver, it would be optimal to use a maximum-likelihood (ML) detector. As the computational effort is of order $M^{n_{T}}$, ML detection is not feasible for real time implementations. Therefore, in the following sections we present suboptimal detection schemes with reduced complexity.

\section{ZERO-FORCING DETECTION}

In this section, different zero-forcing approaches to the estimation of transmit signals in a V-BLAST architecture are reviewed.

\section{A. Linear Zero-Forcing Detector $(Z F)$}

In a linear detector, the receive signal vector $\mathbf{x}$ is multiplied with a filter matrix $\mathbf{G}$, followed by a parallel decision on all layers. Zero-forcing means that the mutual interference between the layers shall be perfectly suppressed. This is accomplished by the Moore-Penrose pseudo-inverse (denoted by $(\cdot)^{+}$) of the channel matrix [7]

$$
\mathbf{G}_{\mathrm{ZF}}=\mathbf{H}^{+}=\left(\mathbf{H}^{H} \mathbf{H}\right)^{-1} \mathbf{H}^{H},
$$

where we assumed that $\mathbf{H}$ has full column rank. The decision step consists of mapping each element of the filter output vector

$$
\tilde{\mathbf{s}}_{\mathrm{ZF}}=\mathbf{G}_{\mathrm{ZF}} \mathbf{x}=\mathbf{s}+\left(\mathbf{H}^{H} \mathbf{H}\right)^{-1} \mathbf{H}^{H} \mathbf{n}
$$

onto an element of the symbol alphabet by a minimum distance quantization. The estimation errors of the different layers correspond to the main diagonal elements of the error covariance matrix

$$
\mathbf{\Phi}_{\mathrm{ZF}}=\mathrm{E}\left\{\left(\tilde{\mathbf{s}}_{\mathrm{ZF}}-\mathbf{s}\right)\left(\tilde{\mathbf{s}}_{\mathrm{ZF}}-\mathbf{s}\right)^{H}\right\}=\sigma_{n}^{2}\left(\mathbf{H}^{H} \mathbf{H}\right)^{-1},
$$

which equals the covariance matrix of the noise after the receive filter. It is obvious that small eigenvalues of $\mathbf{H}^{H} \mathbf{H}$ will lead to large errors due to noise amplification. This effect is especially observed in systems with the same number of transmit and receive antennas. In fact, using a result from random matrix theory [8], it can be shown that in the large system limit for $n_{T}=n_{R} \rightarrow \infty$ the noise amplification tends to infinity almost surely.

\section{B. Zero-Forcing BLAST (ZF-BLAST)}

In [2], a successive interference cancellation technique based on the zero-forcing solution was proposed. Here, the signals are not detected in parallel, but one after another. Assume that layer $i$ yields the smallest estimation error or, equivalently, the largest signal-to-noise ratio (SNR) after linear nulling of the interference. From (4) and (5) it can be concluded that this layer is associated with the row $\mathbf{g}_{\mathrm{ZF}}^{(i)}$ of $\mathbf{G}_{\mathrm{ZF}}$ that has minimum euclidean norm, because this vector causes the smallest noise enhancement. So, during the first step of the algorithm, only the decision statistic

$$
\tilde{s}_{i}=\mathbf{g}_{\mathrm{ZF}}^{(i)} \mathbf{x}=\mathbf{g}_{\mathrm{ZF}}^{(i)}(\mathbf{H s}+\mathbf{n})=s_{i}+\eta_{i}
$$

with the effective noise $\eta_{i}=\mathbf{g}_{\mathrm{ZF}}^{(i)} \mathbf{n}$ is used to find an estimate $\hat{s}_{i}$ for the transmit signal $s_{i}$. The interference caused by this signal is then subtracted from the receive signal vector $\mathbf{x}$ and the $i$-th column is removed from the channel matrix, leading to a new system with only $n_{T}-1$ transmit antennas. This procedure consisting of nulling and cancelling is repeated for the reduced systems until all signals are detected.

Always choosing the layer with the best post detection SNR certainly minimizes the risk of error propagation. Even more, this ordering strategy also maximizes the SNR of the weakest layer in the absence of detection errors and is therefore optimal in the sense of minimum bit error probability [2].

The main computational bottleneck of the originally proposed V-BLAST algorithm is the calculation of the pseudoinverse in each step of detection. This can be avoided using one of the following schemes.

\section{Zero-Forcing BLAST with QR Decomposition (ZF-QRD)}

It was shown in several publications (e.g. [3], [4], [9]) that the BLAST algorithm can be restated in terms of the QR decomposition of the channel matrix $\mathbf{H}$, i.e.

$$
\mathbf{H}=\mathbf{Q R},
$$

where the $n_{R} \times n_{T}$ matrix $\mathbf{Q}$ has orthogonal columns with unit norm and the $n_{T} \times n_{T}$ matrix $\mathbf{R}$ is upper triangular. By multiplying the received signal $\mathbf{x}$ with the hermitian transpose of $\mathbf{Q}$, the sufficient statistic

$$
\tilde{\mathbf{s}}=\mathbf{Q}^{H} \mathbf{x}=\mathbf{R s}+\boldsymbol{\eta} .
$$

for the transmit vector $\mathbf{s}$ is obtained. Note that the statistical properties of the noise term $\boldsymbol{\eta}=\mathbf{Q}^{H} \mathbf{n}$ remain unchanged. Due to the upper triangular structure of $\mathbf{R}$, the $k$-th element of $\tilde{\mathbf{s}}$ is

$$
\tilde{s}_{k}=r_{k, k} \cdot s_{k}+\sum_{i=k+1}^{n_{T}} r_{k, i} \cdot s_{i}+\eta_{k} .
$$

Thus, $\tilde{s}_{n_{T}}$ is free of interference and can be used to estimate $s_{n_{T}}$ after appropriate scaling with $1 / r_{n_{T}, n_{T}}$. Proceeding with $\tilde{s}_{n_{T}-1}, \ldots, \tilde{s}_{1}$ and assuming correct previous decisions, the interference can be perfectly cancelled in each step. Then it follows from (9) that the SNR of layer $k$ is determined by the diagonal element $\left|r_{k, k}\right|^{2}$.

As already mentioned, the detection sequence is crucial due to the risk of error propagation. It can be modified by permuting elements of $\mathbf{s}$ and the corresponding columns of $\mathbf{H}$ prior to the QR decomposition, leading to different matrices $\mathbf{Q}$ and $\mathbf{R}$ [3]. In order to find the optimum sequence, $\left|r_{k, k}\right|$, which represents the length of the component of the column vector $\mathbf{h}_{k}$ that is perpendicular to the space spanned by 
$\mathbf{h}_{1}, \ldots, \mathbf{h}_{k-1}$, needs to be maximized for $k=n_{T}, \ldots, 1$. This may be accomplished in a straight forward way by performing $\mathcal{O}\left(n_{T}^{2} / 2\right)$ different $\mathrm{QR}$ decompositions of permutations of $\mathbf{H}$ [10]. A far more efficient approach is based on the easily verified relation

$$
\mathbf{G}_{\mathrm{ZF}}=\mathbf{H}^{+}=\mathbf{R}^{-1} \mathbf{Q}^{H}
$$

and the fact that the row norms of $\mathbf{G}_{\mathrm{ZF}}$ equal those of $\mathbf{R}^{-1}$. Keeping in mind that the signal $s_{n_{T}}$ is detected first and recalling the optimal ordering criterion from Section III-B, the last row of $\mathbf{R}^{-1}$ must have minimum norm. If necessary, rows of $\mathbf{R}^{-1}$ as well as the corresponding columns of $\mathbf{R}$ have to be exchanged at the expense of destroying the upper triangular structure. However, by right multiplying the permuted version of $\mathbf{R}^{-1}$ with a proper unitary $n_{T} \times n_{T}$ Householder matrix $\Theta$, a block triangular matrix is achieved. Finally, $\mathbf{Q}$ has to be updated to $\mathbf{Q} \Theta$ while the permuted $\mathbf{R}$ is left multiplied with $\Theta^{H}$. These steps are then iterated for the upper left $\left(n_{T}-1\right) \times$ $\left(n_{T}-1\right)$ submatrices of the such modified matrices $\mathbf{R}^{-1}, \mathbf{R}$ and the first $n_{T}-1$ columns of the new matrix $\mathbf{Q}$, resulting in the QR decomposition of the optimally ordered channel matrix H. In [6], a related algorithm that avoids explicit matrix inversions is presented, but the version described here will prove to be more useful later on.

The computational effort is made up of an initial QR decomposition, the inversion of $\mathbf{R}$, and the subsequent ordering, which is dominated by the multiplications of $\mathbf{R}^{-1}, \mathbf{R}$, and $\mathbf{Q}$ with the Householder matrix $\Theta$ in each step. Although this is much better than computing the pseudo-inverse over and over again as in the original ZF-BLAST, a suboptimal algorithm proposed by the authors [3] requiring only a single sorted $\mathrm{QR}$ decomposition is reviewed in the next section.

\section{Zero-Forcing Sorted QR Decomposition (ZF-SQRD)}

In order to obtain the optimal detection order, first $\left|r_{n_{T}, n_{T}}\right|$ has to be maximized over all possible permutations of the columns of the channel matrix $\mathbf{H}$, followed by $\left|r_{n_{T}-1, n_{T}-1}\right|$, and so on. Unfortunately, using standard algorithms for the QR decomposition, the diagonal elements of $\mathbf{R}$ are calculated just in the opposite order, starting with $r_{1,1}$. This makes finding the optimal order of detection such a difficult task.

The sorted QR decomposition (SQRD) algorithm presented in [3] is basically an extension to the modified Gram-Schmidt procedure [11] by reordering the columns of the channel matrix prior to each orthogonalization step. The fundamental idea is that $\left|r_{k, k}\right|$ is minimized in the order it is computed (from 1 to $n_{T}$ ) instead of being maximized in the order of detection (from $n_{T}$ to 1 ). This is motivated by the fact that the layers detected last affect only few other layers through error propagation and may therefore have rather small SNR's, which increases the probability of large SNR's for the first layers. Now, $r_{1,1}$ is simply the norm of the column vector $\mathbf{h}_{1}$, so the first optimization in the SQRD algorithm consists merely of permuting the column of $\mathbf{H}$ with minimum norm to this position. During the following orthogonalization of the vectors $\mathbf{h}_{2}, \ldots, \mathbf{h}_{n_{T}}$ with respect to the normalized vector $\mathbf{h}_{1}$, the first row of $\mathbf{R}$ is obtained. Next, $r_{2,2}$ is determined in a similar fashion from the remaining $n_{T}-1$ orthogonalized vectors, et cetera. Thereby, the channel matrix $\mathbf{H}$ is successively transformed into the matrix $\mathbf{Q}$ associated with the desired ordering, while the corresponding $\mathbf{R}$ is calculated row by row. Note that the column norms have to be calculated only once in the beginning and can be easily updated afterwards. Hence, the computational overhead due to sorting is negligible.

It should be emphasized that SQRD does not always lead to the perfect detection sequence, but in many cases of interest the performance degradation is small compared to the reduced complexity [3]. Whenever SQRD fails to find the optimal order, the algorithm from Section III-C can be applied without having to calculate the initial QR decomposition again. In other words, the computational effort of the optimum algorithm can be decreased by using SQRD to perform a preordering.

\section{MMSE DETECTION}

The problem of noise enhancement through zero-forcing has already been addressed. An improved performance can be achieved by including the noise term in the design of the linear filter matrix $\mathbf{G}$. This is done by MMSE detection schemes, where the filter represents a trade-off between noise amplification and interference suppression.

\section{A. Linear MMSE Detector (MMSE)}

Minimizing the mean squared error (MSE) between the actually transmitted symbols and the output of a linear detector leads to the filter matrix [7]

$$
\mathbf{G}_{\text {MMSE }}=\left(\mathbf{H}^{H} \mathbf{H}+\sigma_{n}^{2} \mathbf{I}_{n_{T}}\right)^{-1} \mathbf{H}^{H} .
$$

The resulting filter output is given by

$$
\tilde{\mathbf{s}}_{\text {MMSE }}=\mathbf{G}_{\text {MMSE }} \mathbf{x}=\left(\mathbf{H}^{H} \mathbf{H}+\sigma_{n}^{2} \mathbf{I}_{n_{T}}\right)^{-1} \mathbf{H}^{H} \mathbf{x}
$$

and, after some manipulations, the error covariance matrix is found to be

$$
\boldsymbol{\Phi}_{\mathrm{MMSE}}=\sigma_{n}^{2}\left(\mathbf{H}^{H} \mathbf{H}+\sigma_{n}^{2} \mathbf{I}_{n_{T}}\right)^{-1} .
$$

With the definition of a $\left(n_{T}+n_{R}\right) \times n_{T}$ extended channel matrix $\underline{\mathbf{H}}$ and $\mathrm{a}\left(n_{T}+n_{R}\right) \times 1$ extended receive vector $\underline{\mathbf{x}}$ through

$$
\underline{\mathbf{H}}=\left[\begin{array}{c}
\mathbf{H} \\
\sigma_{n} \mathbf{I}_{n_{T}}
\end{array}\right] \quad \text { and } \quad \underline{\mathbf{x}}=\left[\begin{array}{c}
\mathbf{x} \\
\mathbf{0}_{n_{T}, 1}
\end{array}\right] \text {, }
$$

the output of the MMSE filter given by (12) can be rewritten as

$$
\tilde{\mathbf{s}}_{\mathrm{MMSE}}=\left(\underline{\mathbf{H}}^{H} \underline{\mathbf{H}}\right)^{-1} \underline{\mathbf{H}}^{H} \underline{\mathbf{x}}=\underline{\mathbf{H}}^{+} \underline{\mathbf{x}} .
$$

Furthermore, the error covariance matrix (13) becomes

$$
\boldsymbol{\Phi}_{\mathrm{MMSE}}=\sigma_{n}^{2}\left(\underline{\mathbf{H}}^{H} \underline{\mathbf{H}}\right)^{-1}=\sigma_{n}^{2} \underline{\mathbf{H}}^{+} \underline{\mathbf{H}}^{+H} .
$$

Comparing (15) and (16) to the corresponding expression for zero-forcing that can be found in (4) and (5), the only difference is that the channel matrix $\mathbf{H}$ has been replaced by $\underline{\mathbf{H}}$. This observation is extremely important for incorporating the MMSE criterion into the previously discussed ZF algorithms. 


\section{B. MMSE-BLAST}

At first sight, the MMSE extension to V-BLAST seems to follow from ZF-BLAST described in Section III-B by simply employing the filter matrix $\mathbf{G}_{\mathrm{MMSE}}$ instead of $\mathbf{G}_{\mathrm{ZF}}$, as proposed in [12]. Although this yields the desired MMSE solution in each detection step, the order is not necessarily optimal. The reason for this is, that the row norms of $\mathbf{G}$ only represent the noise amplification through filtering, which is equivalent to the mean estimation error for zero-forcing. In contrast, after MMSE detection there still remains some residual interference, which, of course, also affects the output signal. Therefore, the layer to be detected must have the largest signal-to-interference-and-noise ratio (SINR), leading to the minimal estimation error. From (16) it follows that this layer corresponds to the row of the pseudo-inverse $\underline{\mathbf{H}}^{+}$with minimum norm. As $\mathbf{G}_{\text {MMSE }}$ consists of the first $n_{R}$ columns of $\underline{\mathbf{H}}^{+}$, the ordering criterion from [12] must be suboptimal.

A straight forward implementation of MMSE-BLAST would calculate the filter $\mathbf{G}_{\text {MMSE }}$ from (11) and the error covariance matrix $\boldsymbol{\Phi}_{\text {MMSE }}$ from (13) in each detection step for the respective reduced system. Fortunately, the similarity of ZF and MMSE detection noted at the end of the last section makes it possible to use the algorithm from Section III-C again. To this end, consider the QR decomposition of the extended channel matrix

$$
\underline{\mathbf{H}}=\left[\begin{array}{c}
\mathbf{H} \\
\sigma_{n} \mathbf{I}_{n_{T}}
\end{array}\right]=\underline{\mathbf{Q}} \underline{\mathbf{R}}=\left[\begin{array}{c}
\mathbf{Q}_{1} \\
\mathbf{Q}_{2}
\end{array}\right] \underline{\mathbf{R}}=\left[\begin{array}{c}
\mathbf{Q}_{1} \underline{\mathbf{R}} \\
\mathbf{Q}_{2} \underline{\mathbf{R}}
\end{array}\right],
$$

where the $\left(n_{T}+n_{R}\right) \times n_{T}$ matrix $\mathbf{Q}$ with orthonormal columns was partitioned into the $n_{R} \times n_{T}$ matrix $\mathbf{Q}_{1}$ and the $n_{T} \times$ $n_{T}$ matrix $\mathbf{Q}_{2}$. Interestingly, the inverse matrix $\underline{\mathbf{R}}^{-1}$ required to find the optimal detection sequence does not need to be calculated explicitly, because from (17) it follows that

$$
\sigma_{n} \mathbf{I}_{n_{T}}=\mathbf{Q}_{2} \underline{\mathbf{R}} \quad \Rightarrow \quad \underline{\mathbf{R}}^{-1}=\frac{1}{\sigma_{n}} \mathbf{Q}_{2},
$$

i.e. the inverse is a byproduct of the initial QR decomposition. This exactly compensates for the additional computational effort due to the additional rows of the extended channel matrix $\underline{\mathbf{H}}$. It seems, that this relation has not been observed before. Furthermore,

$$
\underline{\mathbf{Q}}^{H} \underline{\mathbf{H}}=\mathbf{Q}_{1}^{H} \mathbf{H}+\sigma_{n} \mathbf{Q}_{2}^{H}=\underline{\mathbf{R}}
$$

holds. Using (18) and (19), the filtered receive vector becomes

$$
\tilde{\mathbf{s}}=\mathbf{Q}^{H} \underline{\mathbf{x}}=\mathbf{Q}_{1}^{H} \mathbf{x}=\underline{\mathbf{R}} \mathbf{s}-\sigma_{n}^{2} \underline{\mathbf{R}}^{-H} \mathbf{s}+\mathbf{Q}_{1}^{H} \mathbf{n} .
$$

The second term on the right hand side of (20) including the lower triangular matrix $\underline{\mathbf{R}}^{-H}$ constitutes the remaining interference that can not be removed by the successive interference cancellation procedure.

Since $\mathbf{Q}_{2}$ is proportional to the inverse of $\mathbf{R}$ and $\mathbf{Q}_{1}$ represents the actual filter matrix, the matrices $\mathbf{R}, \mathbf{R}^{-1}$, and $\mathbf{Q}$ encountered in the ZF-QRD algorithm only have to be substituted by $\underline{\mathbf{R}}, \mathbf{Q}_{2}$, and $\mathbf{Q}_{1}$, respectively, in order to get the corresponding optimum MMSE solution.

\section{MMSE Sorted QR Decomposition (MMSE-SQRD)}

Previous publications only treated the sorted QR decomposition in the zero-forcing sense [3], [9]. However, utilizing the extended channel matrix $\underline{\mathbf{H}}$, the results from Section IIID can be adopted to the MMSE case similar to the optimum sorting procedure. Since $\underline{\mathbf{H}}$ initially contains a multiple of the identity matrix in the last $n_{T}$ rows, only the first $n_{R}+k$ rows are considered during the $k$-th step of the MMSESQRD algorithm. This leads to an additional simplification. Furthermore, it ensures the upper triangular structure of $\mathbf{Q}_{2}$.

\section{Performance Analysis}

In the sequel, we investigate the bit error rates (BER) for a MIMO system with $n_{T}=4$ transmit and $n_{R}=4$ receive antennas employing uncoded QPSK modulation. $E_{b}$ denotes the average energy per information bit arriving at the receiver, thus $E_{b} / N_{0}=n_{R} /\left(\log _{2}(M) \sigma_{n}^{2}\right)$ holds. Fig. 2 shows the performance of various zero-forcing detection algorithms and the BER of maximum-likelihood (ML) detection. As expected, the successive detection algorithms outperform the linear ZF detector. The impact of an optimized detection order becomes obvious by comparing the unsorted ZF-QRD, the ZF-SQRD and ZF-BLAST (achieving the optimum detection sequence). ZF-SQRD results in a performance degradation of $1 \mathrm{~dB}$ compared to ZF-BLAST, as it does not always find the optimum order. This degradation reduces for an increasing number of receive antennas, e.g. for a system with $n_{R}=6$ the difference is only $0.5 \mathrm{~dB}$ for a BER of $10^{-5}$ [3].

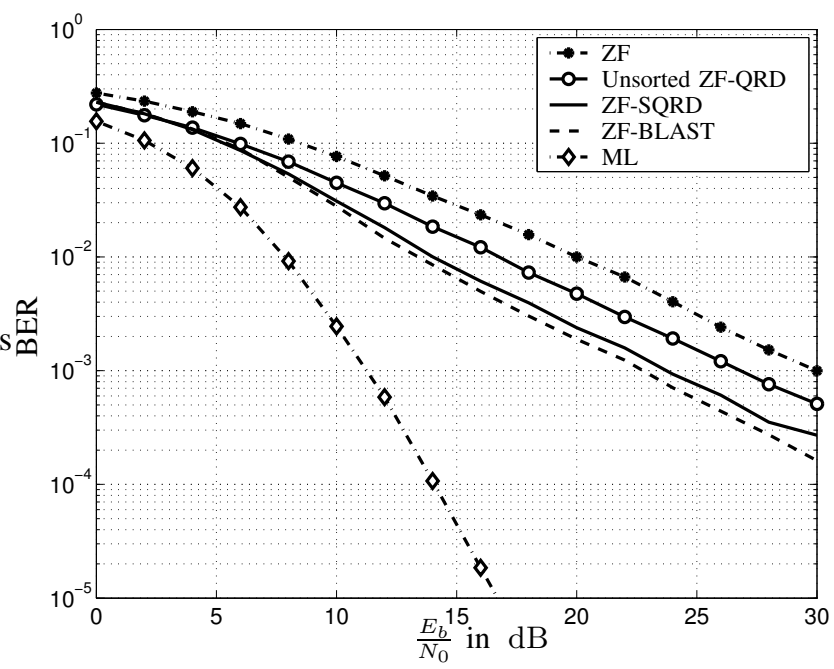

Fig. 2. Simulation with $n_{T}=4$ and $n_{R}=4$ antennas, uncoded QPSK symbols, spectral efficiency of $8 \mathrm{Bit} / \mathrm{s} / \mathrm{Hz}$.

For the same system, Fig. 3 shows the performance of the MMSE detection algorithms. Comparing the simulation results of the successive MMSE detection procedures with the ZFBLAST algorithm, a remarkable performance improvement can be observed. Up to an SNR of $10 \mathrm{~dB}$, the MMSE-SQRD achieves the same performance as the optimal MMSE-BLAST and also outperforms the detection scheme proposed in [12]. In many cases of interest, MMSE-SQRD would be the first 


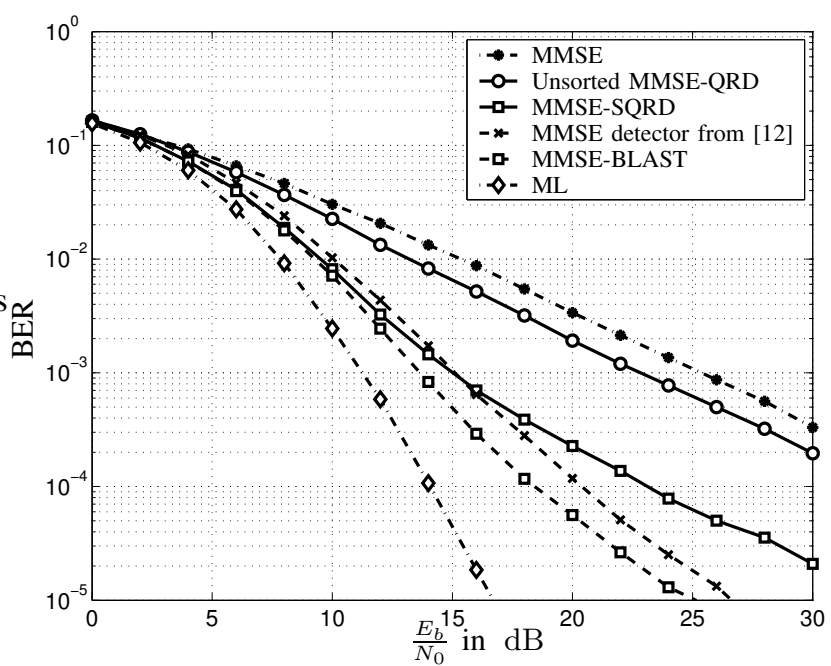

Fig. 3. Simulation with $n_{T}=4$ and $n_{R}=4$ antennas, uncoded QPSK symbols, spectral efficiency of $8 \mathrm{Bit} / \mathrm{s} / \mathrm{Hz}$.

choice for implementation due to the reduced complexity. Note that for the $(4,4)$ system, MMSE-SQRD only fails to find the perfect detection sequence in about $20 \%$ of all channel realizations. In these cases, the optimum ordering strategy can be applied afterwards, thereby closing the increasing performance gap for higher SNR.

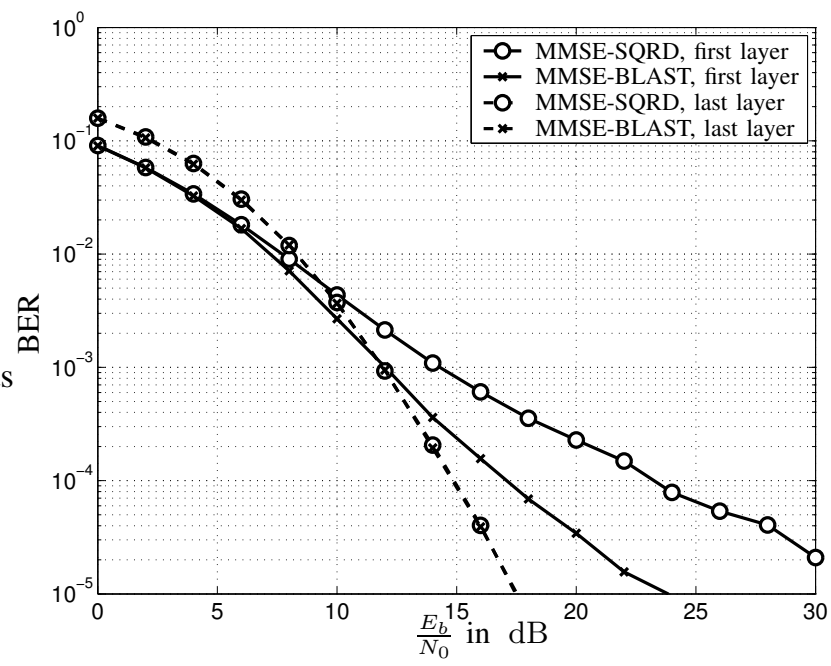

Fig. 4. BER per layer for a $(4,4)$ system without error propagation

In Fig. 4, the BER's of the first and the last layer in the absence of error propagation (genie case) are displayed for MMSE-SQRD and MMSE-BLAST. It can be seen, that the performance degradation is solely caused by the first detected layer, while the BER of the last layer is the same for both schemes. Hence, MMSE-SQRD may be appropriate as an initial stage in iterative schemes.

\section{CONCLUSION}

We have reviewed several detection methods for V-BLAST architectures. First, the ZF criterion was employed. For suc- cessive algorithms, the importance of the detection sequence was pointed out. Based on a QR decomposition of the channel matrix, a way to find the optimal ordering without the need of repeated calculations of pseudo-inverses was described. Additionally, a very efficient sorting strategy proposed by the authors was explained. Using an equivalence relation, these results were adopted to the MMSE criterion, thus leading from the formerly known ZF-SQRD to the new MMSE-SQRD scheme. This algorithm performs a sorted QR decomposition which can be used for subsequent detection. For those cases, where MMSE-SQRD does not find the correct order, a reordering can easily be applied, thereby resulting in an optimum algorithm with reduced complexity.

\section{REFERENCES}

[1] E. Telatar, "Capacity of Multi-antenna Gaussian Channels," European Transactions on Telecommunications, vol. 10, no. 6, pp. 585-595, November-December 2000.

[2] P. W. Wolniansky, G. J. Foschini, G. D. Golden, and R. A. Valenzuela, "V-BLAST: An Architecture for Realizing Very High Data Rates Over the Rich-Scattering Wireless Channel," in Proc. ISSE, Pisa, Italy, September 1998

[3] D. Wübben, J. Rinas, R. Böhnke, V. Kühn, and K. D. Kammeyer, "Efficient Algorithm for Detecting Layered Space-Time Codes," in Proc. ITG Conference on Source and Channel Coding, Berlin, Germany, January 2002, pp. 399-405.

[4] D. Wübben, R. Böhnke, J. Rinas, V. Kühn, and K. D. Kammeyer, "Efficient Algorithm for Decoding Layered Space-Time Codes," IEE Electronic Letters, vol. 37, no. 22, pp. 1348-1350, October 2001.

[5] A. Benjebbour, H. Murata, and S. Yoshida, "Comparison of Ordered Successive Receivers for Space-Time Transmission," in Proc. IEEE Vehicular Technology Conference (VTC), USA, Fall 2001.

[6] B. Hassibi, "An Efficient Square-Root Algorithm for blast," in Proc. IEEE Intl. Conf. Acoustic, Speech, Signal Processing, Istanbul, Turkey, June 2000, pp. 5-9.

[7] S. Verdu, Muliuser Detection, 2nd ed. Cambridge, U.K.: Cambridge University Press, 1998.

[8] J. Silverstein and Z. Bai, "On the Empirical Distribution of Eigenvalues of a Class of Large Dimensional Random Matrices," Journal of Multivariate Analysis, vol. 54, no. 2, pp. 175-192, 1995.

[9] E. Biglieri, G. Taricco, and A. Tulino, "Decoding Space-Time Codes With BLAST Architectures," IEEE Transactions on Signal Processing, vol. 50, no. 10, pp. 2547-2551, October 2002.

[10] G. J. Foschini, G. D. Golden, A. Valenzela, and P. W. Wolniansky, "Simplified Processing for High Spectral Efficiency Wireless Communications Emplying Multi-Element Arrays," IEEE Journal on Selected Areas in Commununications, vol. 17, no. 11, pp. 1841-1852, November 1999.

[11] G. Strang, Linear Algebra and its Applications, 3rd ed. Orlando, Florida: Harcout Brace Jovanovich College Publishers, 1988.

[12] S. Bäro, G. Bauch, A. Pavlic, and A. Semmler, "Improving BLAST Performance using Space-Time Block Codes and Turbo Decoding," in Proc. IEEE Globecom 2000, San Francisco, CA, November 2000. 